\title{
Discrete numerical modeling of loose soil with spherical particles and interparticle rolling friction
}

\author{
Rodaina Aboul Hosn · Luc Sibille · Nadia Benahmed · Bruno Chareyre
}

Published as Aboul Hosn, R., Sibille, L., Benahmed, N. and Chareyre, B. Granular Matter (2017) 19: 4. https://doi.org/10.1007/s10035-016-0687-0

\begin{abstract}
Discrete numerical simulations were carried out to reproduce experimental results obtained on loose cohesionless soil samples subjected to triaxial tests. Periodic boundary conditions were adopted and 3D spherical discrete elements were chosen. However, to overcome excessive rolling of such an oversimplified particle's shape, contact rolling resistance was taken into consideration. The influence of both the elastic and the plastic local parameters is discussed. It is shown that the plastic macroscopic behavior of the granular assembly depends only on the plastic parameters at the microscopic scale, and mainly on the plastic rolling moment reflecting the particle's shape. Moreover, a procedure to obtain an initial density, ranging from loose to dense samples, is proposed by adding adhesion at contacts during the preparation phase. Finally, a calibration procedure is proposed to reproduce experimental results and the limitations of the model are discussed.
\end{abstract}

Keywords Discrete element method · Rolling friction . Calibration $\cdot$ Compaction technique

R. Aboul Hosn

University Grenoble Alpes, 3SR, F-38000 Grenoble, France CNRS, 3SR, F-38000 Grenoble, France

Tel.: +330456528638

E-mail: rodaina.aboulhosn@3sr-grenoble.fr

L. Sibille

University Grenoble Alpes, 3SR, F-38000 Grenoble, France CNRS, 3SR, F-38000 Grenoble, France

N. Benahmed

IRSTEA, Research Unit Recover, 13182 Aix-en-Provence cedex 5, France

B. Chareyre

University Grenoble Alpes, 3SR, F-38000 Grenoble, France CNRS, 3SR, F-38000 Grenoble, France

\section{Introduction}

Most studies based on the discrete (or distinct) element method, pioneered by Cundall and Strack [8], use simplified particle shapes as disks (in 2D) [32] or spheres (in 3D) [31]. Yet, such shapes lead to excessive rolling as compared to real granular materials $[15,1$, 17]. To overcome this setback, complex shapes as polygons [30] and polyhedrons can be used [19]. In fact, such shapes can be complicated and computationally expensive in detecting contacts and calculating forces and torques [24]. Another alternative solution is to use aggregates or clumps of disks or spheres [27]. However, this requires a larger number of spheres and the contribution of the non-convex surface of clumps becomes difficult to evaluate. Thus, one solution is to fix the particle's rotation [4] or to take into account the transfer of a moment between elements in the local constitutive law as pioneered by Iwashita and Oda [22,15,21], or more recently by Estrada [10] to reflect the grain's angularity. In 3D, moment transfer laws can be defined with respect to both twisting (around the contact normal) and rolling (orthogonal to the contact normal) interparticle relative rotation. Only the transfer of rolling moment is addressed in this paper.

Previous studies investigated the influence of the contact rolling resistance properties on the macroscopic behavior $[23,36,35,18]$. All the results showed that increasing the rolling elastic stiffness causes an increase in the macroscopic internal friction angle, but has little influence on the dilatancy $[23,36]$. On the other hand, [35] showed that when the rolling stiffness is high enough, its influence on both the peak friction angle and the dilatancy angle becomes negligible. However, in all cases, 
it is suggested to calibrate the contact rolling stiffness (in addition to the plastic rolling moment) to match the macroscopic internal friction angle. This leads to a surprising cross dependency of microscopic elastic parameters and macroscopic plastic ones. Thereby, the first objective of this paper is to show that such a cross dependency can be avoided.

In addition to the particle's shape and the contact law, the mechanical behavior of a granular material is highly dependent on its initial density. This latter is often reached numerically by playing with the contact friction angle during the compaction phase which proves to be powerful in preparing medium dense to dense samples $[33,6,28]$, but not very loose ones. Indeed, discrete numerical models of loose soils are quite rare in the literature. Hence, the second objective of the paper is to address this issue by adding contact adhesion during the compaction phase of the numerical sample. In this way, we mimic the moist tamping technique used in the laboratory to reconstitute loose soil samples.

Before dealing with these objectives, the contact law including rolling resistance is presented, and the dependency of the stability of explicit numerical integration scheme on the rolling stiffness is defined. Then, a calibration procedure is proposed in the last section by comparing numerical simulations to experimental results of triaxial tests performed on Camargue sandy soil [20,2]. Finally, the limitations of the defined model in reproducing the dependency of the volumetric strains on the mean pressure are discussed.

Note that the effects of some parameters as damping, strain rate or inertial number and tangential stiffness are already known from previous works $[9,7]$ and are not addressed in this paper.

\section{The discrete numerical model}

\subsection{Inter-particle contact law}

Let us consider two spheres in contact with radii $R_{1}$ and $R_{2}$. In the normal and tangential directions to the contact plane, the interaction law is very classical and characterized by constant normal and tangential (or shear) stiffnesses, $K_{n}$ and $K_{s}$, respectively, and a contact friction angle, $\varphi_{c}$, such that:

$$
\begin{aligned}
\vec{F}_{n} & =K_{n} \delta_{n} \vec{n} \\
\Delta \vec{F}_{s} & =-K_{s} \Delta \vec{U}_{s} \quad \text { with } \quad\left\|\vec{F}_{s}\right\| \leq\left\|\vec{F}_{n}\right\| \tan \varphi_{c}
\end{aligned}
$$

where $\vec{n}$ is the normal to the contact plane, $\delta_{n}$, the overlapping distance between spheres, and $\vec{U}_{s}$, the relative tangential (or shear) displacement at the contact point. Only compressive normal forces are modeled, and the contact is lost as soon as the overlap, $\delta_{n}$, vanishes.

The rolling resistance at the contact is defined by the rolling stiffness, $K_{r}$, and the coefficient of rolling friction, $\eta_{r}$ (it is commonly called friction because of the mathematical form it takes although it doesn't directly involve the friction between two surfaces). Thus, the rolling moment, $\vec{M}_{r}$, acting against the relative rolling rotation of particles, $\vec{\theta}_{r}$, is expressed as:

$\Delta \vec{M}_{r}=-K_{r} \Delta \vec{\theta}_{r} \quad$ with $\quad\left\|\vec{M}_{r}\right\| \leq\left\|\vec{F}_{n}\right\| \eta_{r} \min \left(R_{1}, R_{2}\right)$

$\Delta \vec{\theta}_{r}$ is computed as the tangential component of the incremental relative rotation, $\Delta \vec{\theta}$, of the contacting spheres. For spheres with incremental rotations, $\Delta \vec{\omega}_{1}$ and $\Delta \vec{\omega}_{2}$ respectively, $\Delta \vec{\theta}=\Delta \vec{\omega}_{2}-\Delta \vec{\omega}_{1}$ and:

$\Delta \overrightarrow{\theta_{r}}=\Delta \vec{\theta}-(\Delta \vec{\theta} \cdot \vec{n}) \vec{n}$

Note that Equation (3) defining the contact resistance to rolling is similar to Equation (2) representing the sliding resistance due to dry friction. Therefore, contact resistance to rolling can be interpreted as a rolling friction.

To avoid any dependency of macroscopic elastic properties on particle size, contact stiffnesses are defined from a stiffness modulus, $E_{c}$, and dimensionless shear and rolling coefficients, $\alpha_{s}$ and $\alpha_{r}$, respectively:

$K_{n}=2 E_{c} \frac{R_{1} R_{2}}{R_{1}+R_{2}} ; \quad K_{s}=\alpha_{s} K_{n} ; \quad K_{r}=\alpha_{r} R_{1} R_{2} K_{s}$.

Finally, adhesive normal and tangential forces, $F_{n}^{A}$ and $F_{s}^{A}$, can be added to the contact law (used here during the sample compaction only) and defined from an adhesive stress $\sigma_{A}$ such that:

$F_{n}^{A}=F_{s}^{A}=\sigma_{A}\left[\min \left(R_{1}, R_{2}\right)\right]^{2}$

Then, the contact presents a resistance to a tensile normal force as long as:

$\vec{F}_{n} \cdot \vec{n}>-F_{n}^{A}$,

and a maximum shear force against sliding is given as:

$\left\|\vec{F}_{s}\right\| \leq\left\|\vec{F}_{n}\right\| \tan \varphi_{c}+F_{s}^{A}$. 
2.2 Stability condition of the explicit integration scheme

The stability condition of the explicit centered finite difference scheme, integrating motion equations of particles, can be derived by determining a stiffness matrix $\mathbf{K}$, linking displacement and rotation of an inertial particle to force and torque $[12,6]$. $\mathbf{K}$ refers to a particular contact network, thus changes during the simulation. The full details of the derivations are given in the Appendix. In order to understand the role of each stiffness in the stability condition, we consider the following simplifying assumptions (not assumed in the numerical model):

1. all spheres are identical (same size and same inertia)

2. all contacts have the same stiffness values

3. the stiffness tensors are isotropic.

The small movements $\vec{u}$ and $\vec{\theta}$, in translation and in rotation, respectively, of a particle relative to an initially stable configuration are described by (see Appendix):

$\vec{u}=-\frac{N_{c} K_{n}\left(1+2 \alpha_{s}\right)}{M} \vec{u}$,

$\overrightarrow{\ddot{\theta}}=-\frac{N_{c} K_{n}\left(5 \alpha_{s}+5 \alpha_{r n}+2.5 \alpha_{t w}\right)}{M} \vec{\theta}$

where $\alpha_{r n}$ and $\alpha_{t w}$ are respectively the rolling and twisting coefficients (defined in Appendix), $K_{n}$ is the normal stiffness, $M$ is the mass of the particle and $N_{c}$ is the number of contacts formed by the particle. Numerators in the right hand sides of the uncoupled equations of motion 9 and 10 represent the global stiffnesses of the particle in translation and rotation respectively. The critical time step will be fixed by the highest global stiffness. So how the different kinds of contact stiffnesses can affect the order of magnitude of the timestep?

- if the normal stiffness is dominant $\left(\alpha_{k} \ll 1\right.$ for all $k$ ), the stability condition is controlled by the translational motion (eq. (9));

- if $\alpha_{s}$ is not negligible, precisely as soon as $\alpha_{s} \geq 1 / 3$, the rotational motion governs the stability condition (eq. (10));

- if $\alpha_{r n} \geq 1 / 3$ or $\alpha_{t w} \geq 2 / 3$ (even if $\alpha_{s}$ remains small), the rotational motion governs the stability condition;

- if $\alpha_{k} \approx 1$ for all $k$, the timestep imposed by the rotational motion is less than half of the one corresponding to translational motion

- if $\alpha_{k}>1$ for at least one $k$, estimating the timestep from $k_{n}$ alone leads to overestimate the maximum allowed timestep significantly.
When the numerical scheme is unstable due to the translational motion, particles are usually ejected away from the granular mass after few timesteps. In most cases it cannot remain unnoticed by the user, so that mistakes on the timestep are immediately detected. On the other hand, preliminary numerical experiments (not reported here) showed that the consequences of numerical instabilities for rotational modes may not be obvious at the macroscale. Only more detailed examinations may reveal spurious relative motion at some contacts and high angular velocities of few particles. It is thus highly required to use a robust and validated procedure for choosing the timestep in the general case. The numerical results may otherwise suffer from (apparently) unexplained bugs. The fact that this problem has not been clearly described previously may explain why the stiff limit for contact moments was not shown by most previous authors.

\section{Macroscopic constitutive behaviour}

Numerical triaxial compression tests were performed using YADE software [34] to investigate the effects of some local mechanical parameters on the constitutive behavior of the model. It was made up of 10,000 spherical discrete particles whose radius is equal to 0.014 and 0.026 to avoid crystallization. Periodic boundary conditions were adopted. The periodic cell was formed as a parallelepipedic block filled with a cloud of spheres (i.e. assembly of nonoverlapping particles). The non-overlap constraint necessarily leads to rather loose clouds. Since this is only the starting point of the compaction, it has no consequences on the equilibrium state after compaction. Then, isotropic compaction was applied until reaching the required confining pressure $P_{c}$. Finally, the triaxial compression was performed. During the compaction phase, the stress is controlled to reach a confined pressure of $80 \mathrm{kPa}$. In the triaxial compression phase, the inertia number is chosen $1.65 \times 10^{-4}$ to avoid inertial effects. In this work, the timestep was chosen to be $80 \%$ of the maximum value defined by the stability condition (eq. 20 in appendix). Moreover, no damping is considered in the triaxial phase. Typical simulated responses can be seen in Figures 3 and 5. Table 1 summarizes the parameters used for these studies.

\subsection{Elastic local parameters and plastic macroscopic properties}

In this section, the influence of elastic local parameters, such as the normal and the rolling stiffnesses, on the macroscopic shear strength will be examined. Shear 


\begin{tabular}{|l|l|l|l|l|l|l|l|l|}
\hline & $\begin{array}{l}P_{c} \\
\mathrm{kPa})\end{array}$ & $\begin{array}{l}E_{c} \\
(\mathrm{kPa})\end{array}$ & $\kappa$ & $\begin{array}{l}\alpha_{s} \\
(\mathrm{deg})\end{array}$ & $\varphi_{c}$ & $\alpha_{r}$ & $\begin{array}{l}\eta_{r} \\
(\mathrm{kPa})\end{array}$ & $\begin{array}{l}\sigma_{A} \text { (for com- } \\
\text { paction only) }\end{array}$ \\
\hline Case 1 & 80 & $810^{2}-810^{7}$ & $50-510^{5}$ & 0.8 & 30 & - & - & - \\
\hline Case 2 & 80 & $310^{5}$ & 1875 & 0.8 & 30 & $0.025-2.5$ & $0.01-5$ & - \\
\hline Case 3 & 80 & $310^{5}$ & 1875 & 0.8 & $5-40$ & 1.25 & 0.1 & - \\
\hline Case 4 & 80 & $310^{5}$ & 1875 & 0.2 & 30 & 5 & 0.1 & $0-200$ \\
\hline
\end{tabular}

Table 1: Summary of the parameters used in each case of the parametric studies.

strength at failure is characterized by the peak friction angle, $\varphi_{p}$, reached at the peak of the deviator stress, $q$, along a drained compression (constant effective confining stress), while, $\varphi_{0}$, characterizes the shear strength at the residual state (or the critical state).

We investigate first the effect of the normal contact stiffness represented via the dimensionless stiffness $\kappa$ :

$\kappa=\frac{E_{c}}{2 P_{c}}$

The response shown in Figure 1, where no rolling resistance was introduced (case 1 in Table 1), demonstrates that the peak friction angle, $\varphi_{p}$, decreases with the normal contact stiffness particularly for $\kappa<50$. Similar results appear in $[25,26]$. This is a remarkable disagreement with the experimental results on sand, in which the peak friction angle decreases for decreasing $\kappa$ (i.e. for increasing the contact stiffness). This experimental trend may result simply from the dependency of the contact friction on the normal force [29]. In numerical models, the contact friction coefficient is usually constant. Thus the decreasing trend of the internal friction is explained by the decreasing number of contacts. Moreover, Figure 1 shows that for a higher dimensionless stiffness, where the overlap becomes negligible, there is still a scattering among the values of $\varphi_{p}$, but quite low (about $\pm 0.5^{\circ}$ around the mean value). Thereby, for $\varphi_{c}=30^{\circ}$, plastic failure seems not significantly dependent of the contact normal stiffness for a value of $\kappa$ greater than 50 .

A more extended parametric study was performed concerning the parameters involved in the contact resistance to rolling. The values of the parameters defined in Table 1, case 2, were chosen in order to test different pairs of $\alpha_{r}$ and $\eta_{r}$ (note that for isotropic shape particles, $\eta_{r}$ should be $\leq 1$ [15]. However, $\eta_{r}>1$ are investigated in this preliminary section). Macroscopic shear strength in terms of peak, $\varphi_{p}$, and residual, $\varphi_{0}$, friction angles is shown in Figure 2. Note that the residual

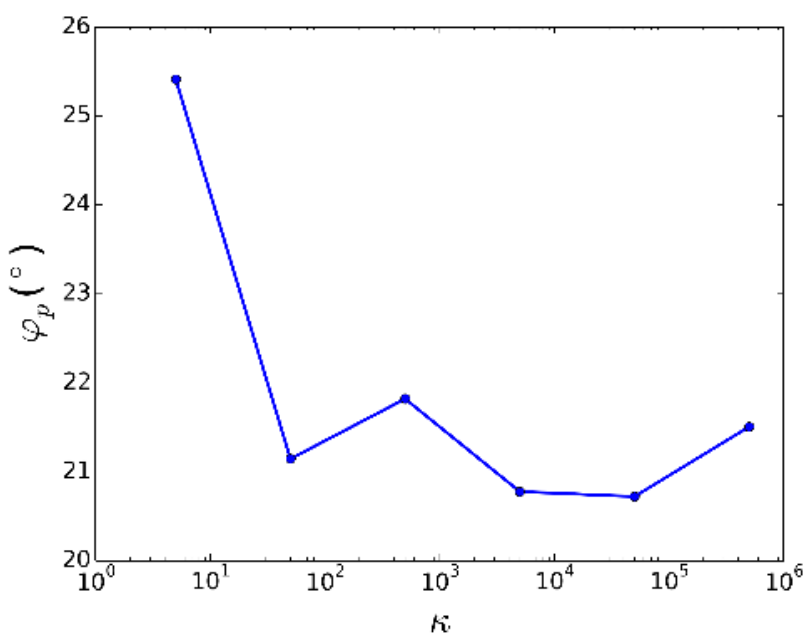

Fig. 1: The influence of the reduced contact stiffness $\kappa$ on the peak friction angle (case 1 in Table 1 ).

friction angle is calculated from the average of stresses corresponding to $\epsilon_{a}>25 \%$. The results demonstrate that for a rolling stiffness, $\alpha_{r}$, sufficiently high (here for $\alpha_{r} \geq 1.25$ ), the macroscopic plastic parameters reach constant values which depend only on $\eta_{r}$ [35].

Thus, if the contacts are sufficiently stiff, then the microelastic parameters, including rolling stiffness, have no influence on macro-plastic parameters. Approaching these normal and rolling stiff limits, should always be preferred to avoid cross dependencies between macroscopic plastic properties and elastic local parameters, in contrast with what was suggested in $[23,36]$.

\subsection{Plastic local parameters and plastic macroscopic} properties

The influence of the plastic local parameters, $\eta_{r}$ and $\varphi_{c}$, on the plastic macroscopic properties can be analyzed independently from the local elastic stiffnesses (as 


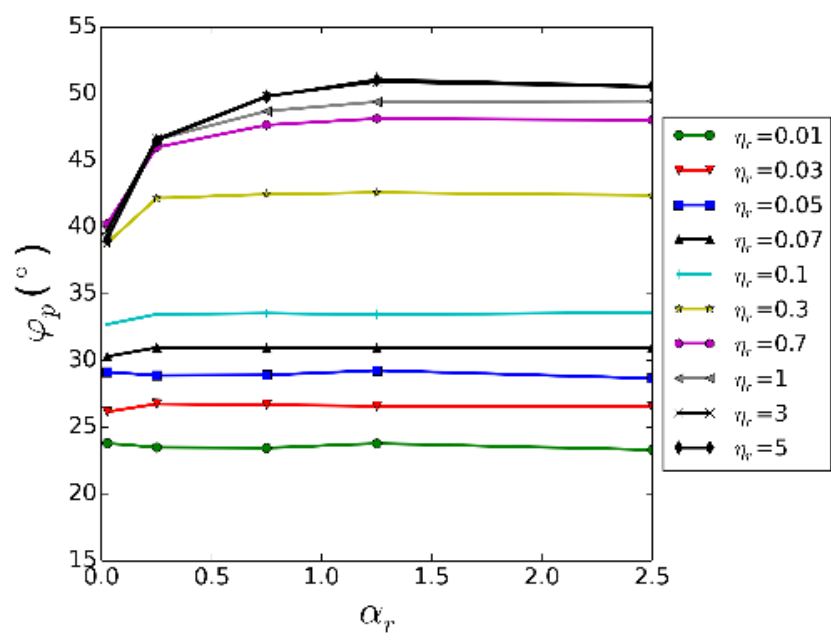

(a)

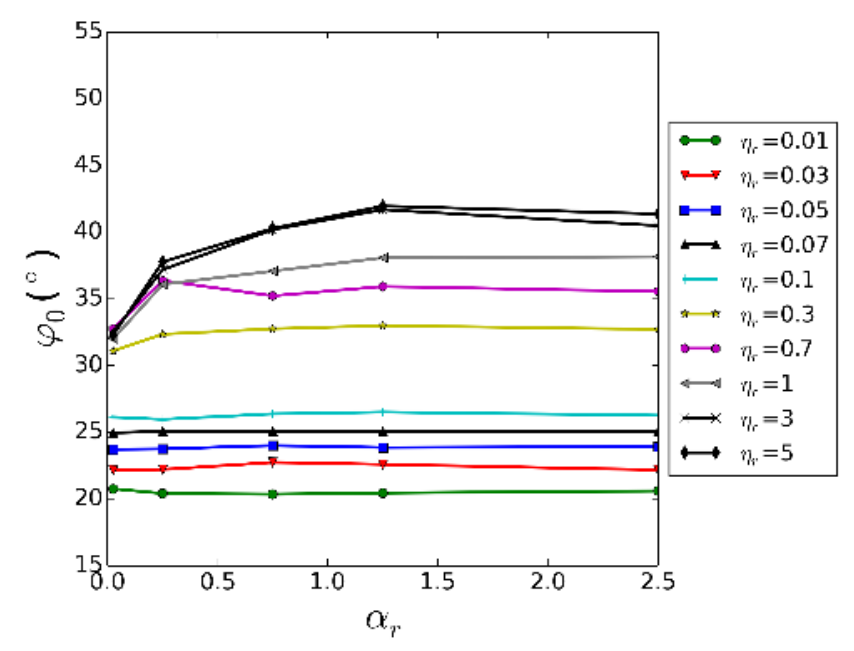

(b)

Fig. 2: The variation of (a) peak friction angle, $\varphi_{p}$, and (b) residual friction angle, $\varphi_{0}$, with respect to $\alpha_{r}$ for different values of $\eta_{r}$ (case 2 in Table 1 ).

long as the elastic stiffnesses are beyond the stiff limits). Therefore, $E_{c}$ is set in the following to $310^{5} \mathrm{kPa}$ $(\kappa=1875)$ and $\alpha_{r}$ to 1.25 . In addition, only cases for $\eta_{r}$ $\leq 1$ are considered. Figure 3 shows the variation of the stress ratio $\left(\frac{q}{p}\right)$ and porosity ( $\mathrm{n}=\frac{V_{v}}{V_{t}}$, where $V_{v}$ is the volume of voids and $V_{t}$ is the total volume of the sample) versus axial deformation during a triaxial compression for different $\eta_{r}$ values. As can be seen, both peak and residual shear strengths depend on $\eta_{r}$, as well as on the dilatancy. The contact resistance to rolling increases with $\eta_{r}$, mimicking more and more angular particles, leading to higher peak and residual friction angles and to more dilatant behaviors. This is also shown in Fig- ure 4 where the peak shear strength and the residual shear strength depend only on rolling friction, $\eta_{r}$, for sufficiently high values of $\alpha_{r}$ (i.e. without the effect of an elastic parameter).

On the other hand, a series of triaxial compressions were simulated with different values of the contact friction angle (Table 1, case 3). The response of the model is illustrated in Figure 5. It is shown that this parameter has a strong effect on the peak strength and dilatancy. However, for the initial porosity considered, only dilatant behaviors are observed, and purely contractant responses are not reproduced even for low values of $\varphi_{c}$. Finally, for $\varphi_{c}>15^{\circ}$, its influence on the residual shear strength becomes insignificant, as observed similarly by [14].

Thus, for $\varphi_{c}>15^{\circ}$, the residual shear strength is governed only by $\eta_{r}$, whereas the peak strength can be fine tuned by $\varphi_{c}$ for a fixed value of $\eta_{r}$. Volumetric strain cannot be adjusted with $\varphi_{c}$ and/or $\eta_{r}$ alone since, for instance, it was not possible to reach a contractant behavior during the parametric studies displayed in Figures 3 and 5. Nevertheless, the volumetric strain is also strongly dependent on the initial density, and a purely contractant behavior may be observed for loose or very loose granular materials. Consequently, a numerical preparation methodology allowing to reach a wide range of the initial porosity of the model is presented in the next section. 3.3 Preparation methodology for a large range of
initial density

In the literature, several techniques to generate numerical discrete samples are suggested. They mainly include the isotropic-compression method [8] and the particle expansion method [23,35]. Both methods can be combined with a progressive lubrication of contacts consisting in decreasing the inter-particle friction until reaching the target porosity (as proposed for instance in [5, 33]). Such methods are efficient means to produce dense samples but they fail in repoducing very loose ones. Alternatively, the multi-layer with under-compaction method proposed in [16] is capable of generating homogeneous samples with a variety of density conditions ranging from very loose to dense states. It is based on dividing evenly the sample into several layers, each compacted to a state looser that the upper one. However, little studies as the latter have been done on loose samples.

We propose thereafter a new method for that purpose. 


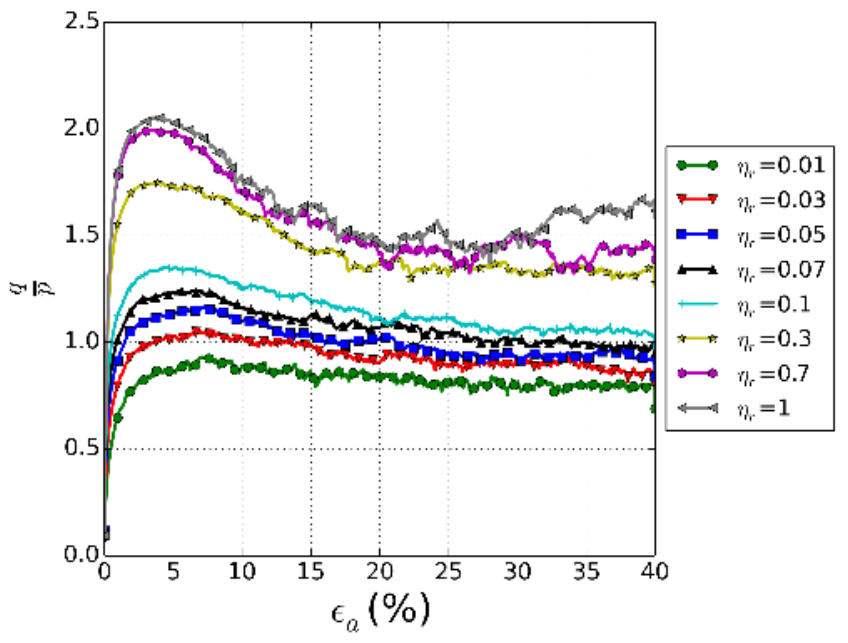

(a)

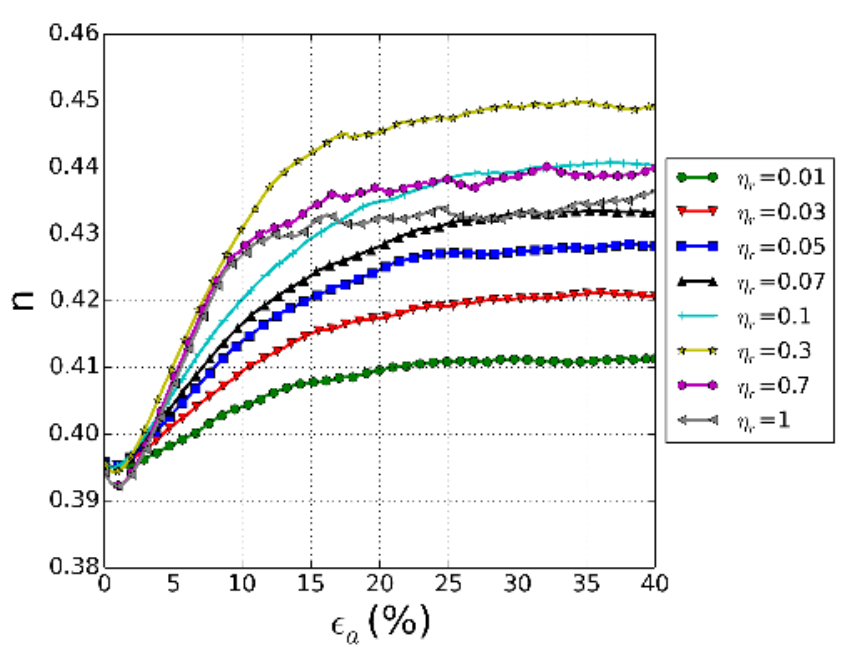

(b)

Fig. 3: The variation of (a) the stress ratio and (b) porosity during triaxial drained compressions under different values of $\eta_{r}$ (case 2 in Table 1 for $\alpha_{r}=1.25$ ).

It imitates numerically the experimental reconstitution technique of soil samples by the moist tamping method where a low moist content (about 2 to $3 \%$ ) introduces adhesion between particles allowing to reach even higher void ratios from the ones corresponding to the loosest state achieved by [16]. Moreover, as explained in [11], introducing cohesion in the model, in the presence of rolling resistance, stabilizes looser and less coordinated samples leading to a reduction in the solid fraction and coordination number.

The methodology comprises three steps as follows:

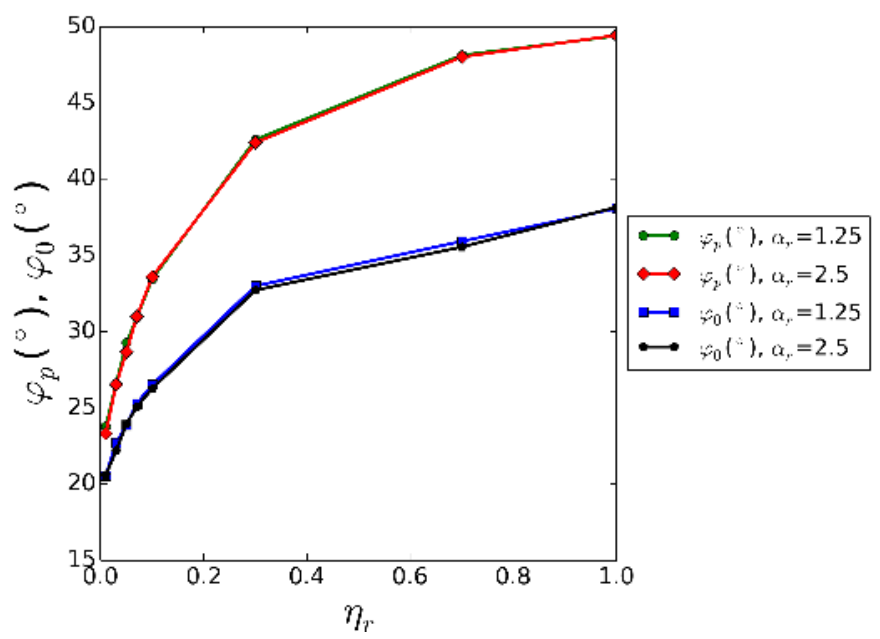

Fig. 4: The variation of peak and residual friction angles with respect to $\eta_{r}$ for different values of $\alpha_{r}$ (case 2 in Table 1 for $\alpha_{r}=1.25$ and 2.5).

1. Random generation of a very loose cloud of nonoverlapping particles.

2. Isotropic compaction until a target confining pressure is performed with the presence of an adhesive contact stress, $\sigma_{A}$ (Eq. 6) and keeping the normal contact friction angle (i.e. the same value used later for compression tests), first at a high strain rate to save computational time, then slower when contacts start to percolate, until equilibrium is achieved (see Figure 6).

3. Removal of the contact adhesion $\left(\sigma_{A}=0\right)$ and waiting for a new equilibrium to be attained.

Figure 6 shows the evolution of the porosity and the unbalanced forces in each step. It can be seen that during the compaction process that the porosity decreases until an equilibrium state is reached. Equilibrium is assessed from the dimensenionless unbalanced force, $U_{f}$. It is computed as the ratio of the mean resultant particle force to the mean contact force. $U_{f}$ tends to zero for a perfect static equilibrium and a value of $U_{f}=10^{-3}$ was considered here as representative of a state sufficiently close to this limit (Figure 6). Notice that during the last step where contact adhesion is removed, the sample experiences a slight additional compaction (see Figure 6), as observed experimentally with the moist tamping technique during the saturation stage of the soil sample.

Following the proposed procedure, the accessible densities reached by this method are shown in Figure 7 . It can be noticed that as the contact adhesion, $\sigma_{A}$, in- 


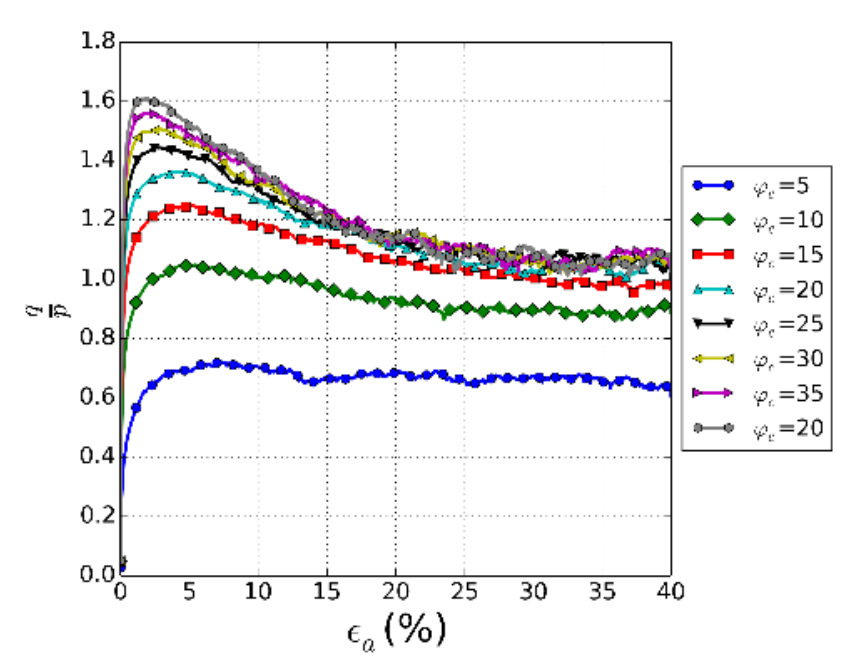

(a)

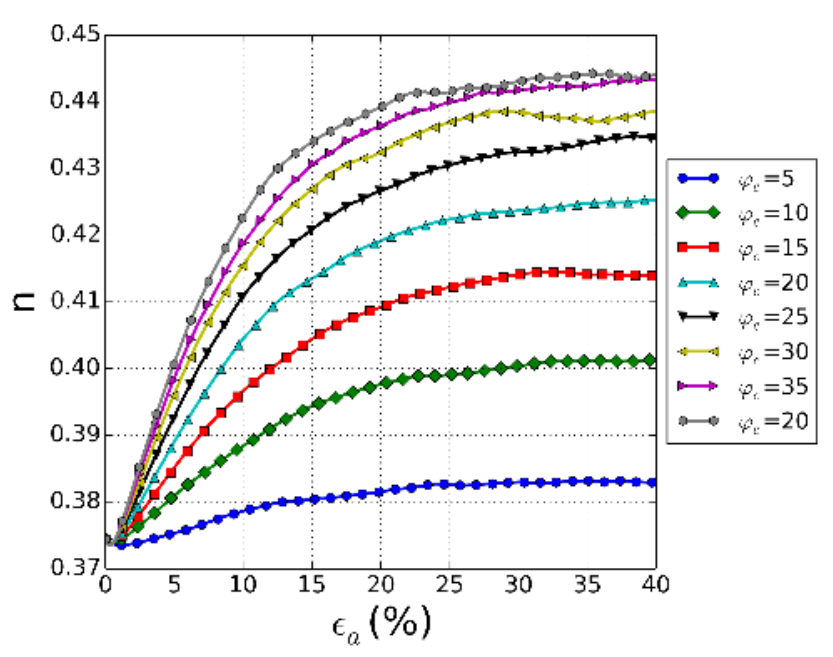

(b)

Fig. 5: The variation of (a) the stress ratio and (b) porosity during triaxial compressions under different values of $\varphi_{c}$ (case 3 in Table 1 ).

creases, the initial porosity after compaction increases too, and the sample shows more and more an obvious contractive behavior as shown in Figure 8. This happens up to a threshold adhesion value (here equal to $\sigma_{A}=80 \mathrm{kPa}$ or $\frac{\sigma_{A}}{P_{c}}=1$ ), beyond which the numerical sample strongly collapses during the third step of the compaction method leading to lower porosities and more dilatant behavior (see the results for $\frac{\sigma_{A}}{P_{c}}=1.25$ and $\frac{\sigma_{A}}{P_{c}}=2.5$ in Figures 7 and 8 ). Figure 8 shows the triaxial compressions simulated from the accessible densities reached by this method. It can be observed that removing completely the adhesion during the compaction phase is not enough to reach dense samples presenting a strong dilatant behavior. For that, contacts need to

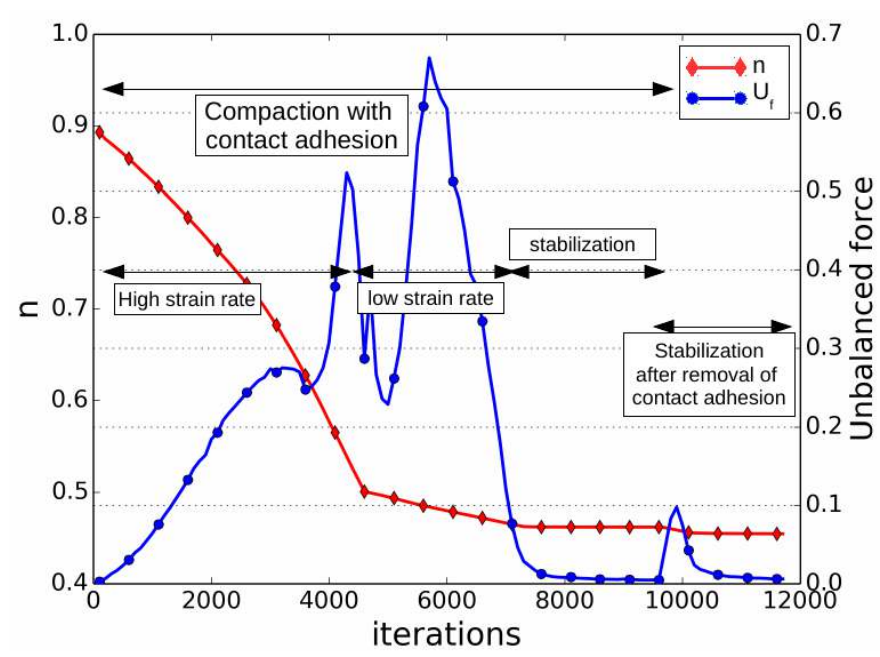

Fig. 6: Evolution of the porosity and the dimensionless unbalanced force during the three steps of compaction.

be lubricated during the compaction phase by fixing a low friction angle, as described for instance in [33], and illustrated in Figures 7 and 8 for a lower values of the contact friction angle during compaction. Hence, a large range of initial density can be reached by assigning during compaction either an artificial contact adhesion or a reduced contact friction.

\section{Modelling of the soil mechanical behaviour}

In this section, a calibration procedure is defined and then applied to reproduce the experimental data obtained from drained triaxial compression tests with $P_{c}=$ $200 \mathrm{kPa}$ (i.e. constant effective confining stress). Moreover, to validate our model, it was tested with the data obtained from: triaxial compression tests with different confining pressures, undrained triaxial test (i.e. no volume change) as well as constant deviator stress test (constant q) [20].

\subsection{Calibration process}

After studying the influence of different parameters incorporated in our DEM model, a calibration procedure based on the simulated response to a triaxial compression was defined as follows:

1. As the residual shear strength is independent of the initial porosity and of the contact friction for $\varphi_{c}>15^{\circ}$, then $\eta_{r}$ can be first calibrated by reproducing the experimental residual friction angle while $\varphi_{c}$ is set arbitrarily (let's say for instance $30^{\circ}$ 


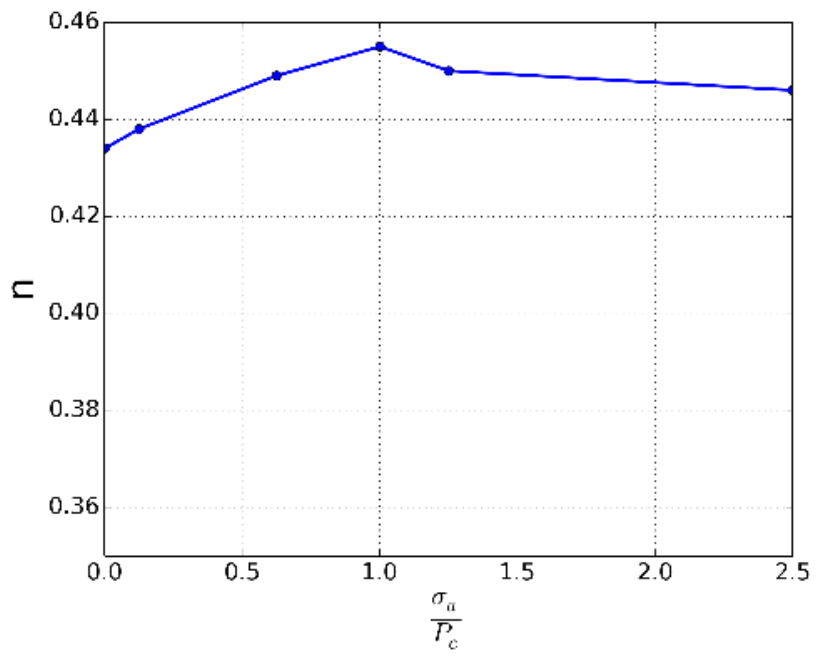

(a)

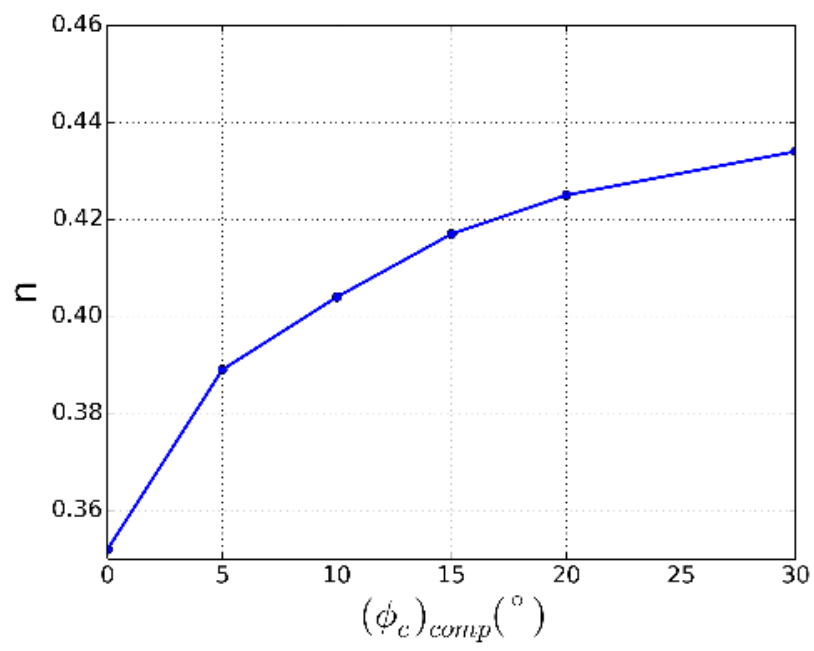

(b)

Fig. 7: Range of porosity reached after compaction for: (a) introduction of contact adhesion $\sigma_{a}$ during compaction (with $\left(\phi_{c}\right)_{c o m p}=30^{\circ}$ ), and (b) reduction of contact friction angle, $\left(\phi_{c}\right)_{c o m p}$, during compaction.

as a first approximation of the contact friction between two silica particles). For this step, the numerical sample may be compacted without the addition of contact adhesion or contact lubrication in order to start from a medium dense material.

2. Volumetric strain (i.e. dilatancy / contractancy) and peak stress (if any) are approached as close as possible by fine tuning the initial sample density. To get a looser material, adhesion is introduced during the compaction phase, while contacts are lubricated during the latter phase to reach a denser assembly, as described in the previous section.

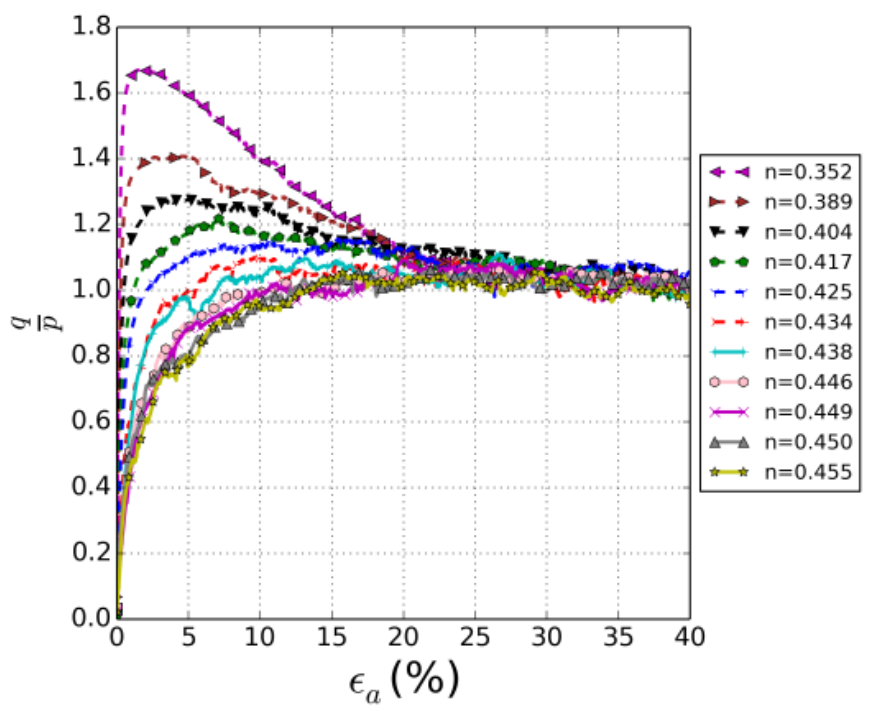

(a)

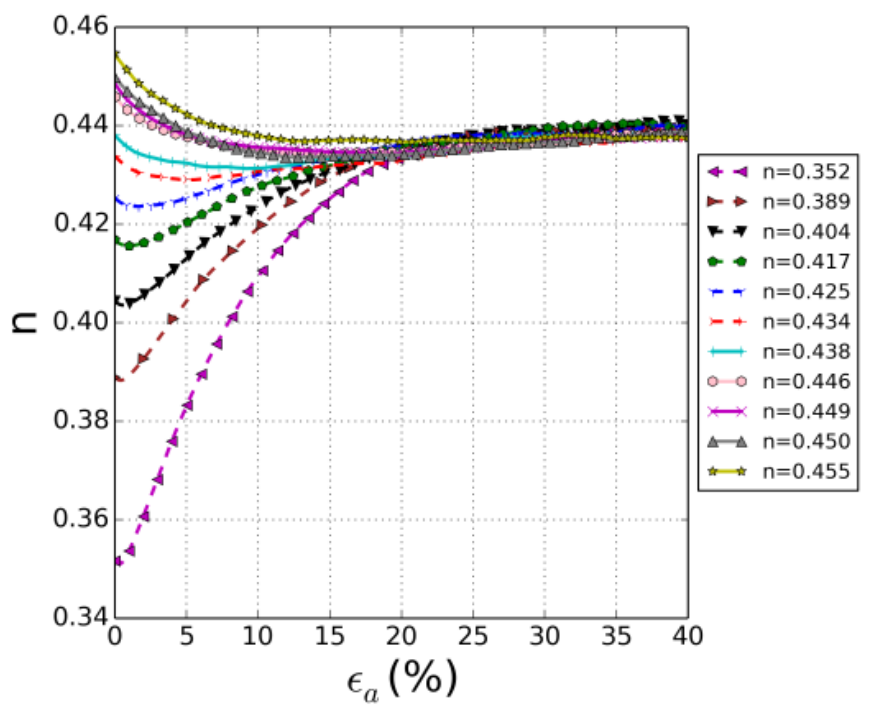

(b)

Fig. 8: Effect of adhesion, $\sigma_{A}$, during compaction, on (a) the stress-strain response to a triaxial compression and (b) porosity (case 4 in Table 1 ).

3. Contact friction angle $\varphi_{c}$ is calibrated (if necessary) to improve the reproduction of the volumetric strain and the peak stress. Knowing that a change of $\varphi_{c}$ can affect the initial porosity reached after the compaction phase, it may be necessary to reiterate steps 2 and 3 until a satisfying calibration of both the initial porosity and $\varphi_{c}$ is achieved.

Calibration of elastic parameters, contact elastic modulus, $E_{c}$, and shear factor, $\alpha_{s}$, appears secondary to plastic parameters (as long as they are beyond the stiff 
limit), with respect to their effects on the macroscopic mechanical behavior. They can be adjusted if necessary to better reproduce the macroscopic initial Young modulus and Poisson ratio as discussed in [23]. Rolling stiffness, $\alpha_{r}$, is just kept close to the rigidity limit, not lower to avoid any impact on plastic macroscopic properties, but not higher to avoid possible reduction of the critical time step as discussed in section 2.2.

It is worth noting that this proposed calibration procedure stresses more on the rolling friction than on the contact friction angle. Indeed, one can expect the contact friction angle not to be very different for different granular soils since grains are generally made with similar constituents (silica and alumine), whereas particle shapes, reflected by the rolling friction, may change a lot and directly impact the soil mechanical behavior.

\subsection{Validation on laboratory triaxial compression paths}

The calibration procedure is applied to calibrate the numerical model on experimental data obtained from drained triaxial compression tests on a Camargue sandy soil. Laboratory triaxial compression results are presented in $[20,2]$. The triaxial tests were performed on very loose Camargue sand samples prepared with an initial (i.e. before isotropic compression) relative density of $20 \%$. The particle size distribution of the numerical model partially follows the one of the sand as presented in Figure 9. It has been simplified by removing $3 \%$ of the largest particles and $3 \%$ of the smallest ones to limit the total number of discrete elements and so to reduce the computational cost.

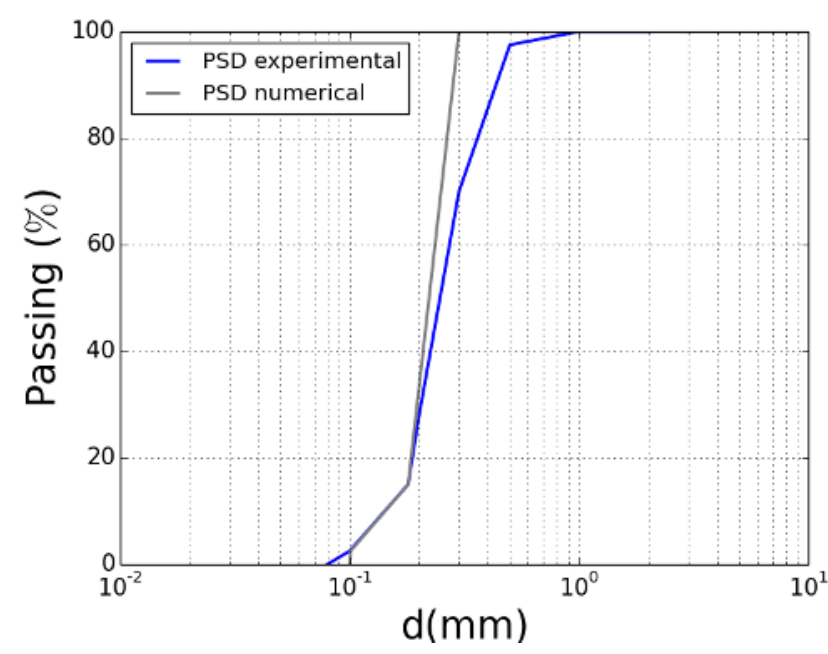

Fig. 9: The gradation curve of the Camargue sand.
The model has been calibrated from a drained triaxial compression with a confining pressure, $P_{c}=200 \mathrm{kPa}$ (Figure 10), leading to the parameters shown in Table 2 .

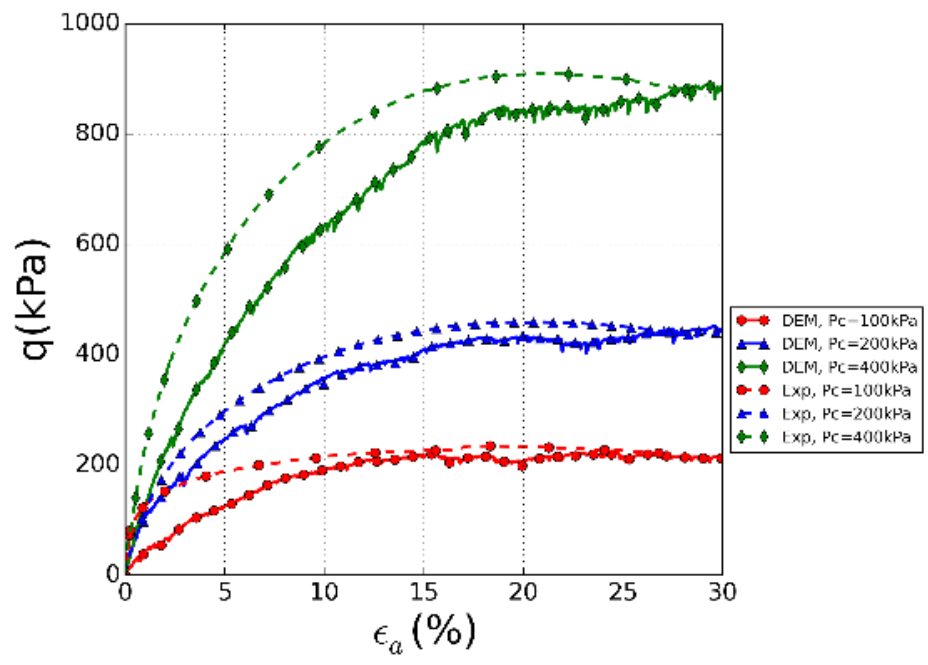

(a)

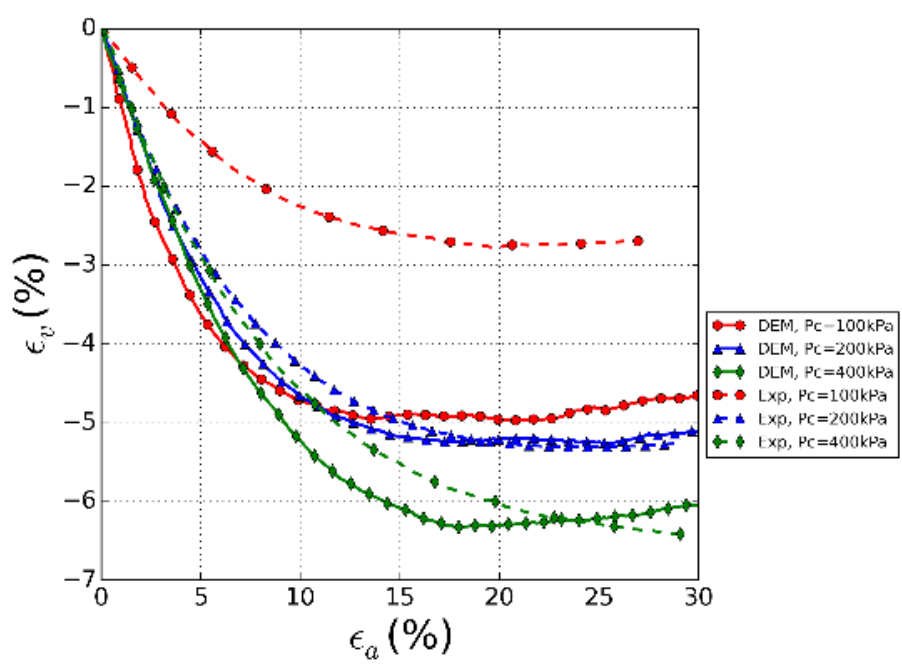

(b)

Fig. 10: Comparison between experimental drained triaxial compression tests and DEM simulations on loose Camargue sand. (a) deviator stress, (b) volumetric strain.

When applied to compressions with different confining pressures than the one used for calibration (Figure 10), the model succeeded to describe the shear strength but partially failed in reproducing quantitatively the dependence of the volumetric strain on the confining pressure. The important reduction of contractancy of the sand obtained experimentally for $P_{c}=100 \mathrm{kPa}$, with respect 


\begin{tabular}{|l|l|l|l|l|l|l|}
\hline $\begin{array}{l}P_{c} \\
(\mathrm{kPa})\end{array}$ & $\begin{array}{l}E_{c} \\
(\mathrm{kPa})\end{array}$ & $\alpha_{s}$ & $\begin{array}{l}\varphi_{c} \\
(\mathrm{deg})\end{array}$ & $\alpha_{r}$ & $\eta_{r}$ & $\begin{array}{l}\sigma_{A} \text { (for com- } \\
\text { paction only) } \\
(\mathrm{kPa})\end{array}$ \\
\hline 200 & $210^{5}$ & 0.2 & 25 & 7.5 & 0.22 & 200 \\
\hline
\end{tabular}

Table 2: Summary of the parameters calibrated on the loose Camargue sand.

to $P_{c}=200 \mathrm{kPa}$ and $400 \mathrm{kPa}$, is underestimated by the model.

Responses of the calibrated model and of the sand subjected to an undrained compression, after an initial isotropic compression, $P_{c}=200 \mathrm{kPa}$, are compared in Figure 11. The model exhibits sudden and total liquefaction at very low axial strain which was not the case experimentally for the sand, as if the ability of the numerical model to contract was more important than for the sandy sample. Responses were also compared to a constant deviator stress loading path performed after an initial drained compression. Stress and strain paths are displayed in Figure 12. The numerical model correctly describes the stress state at failure occurrence but predicts a much more contractant response than the experimental data. The constant shear drained path, such as the $q$-constant path, lead to a reduction of the mean pressure $p$. Consequently, the overestimation of contractancy along these loading paths is in agreement with the results obtained from the drained compressions (Figure 10). In the latter case, we showed that the model underestimates the reduction of contractancy with the decrease of the mean pressure.

The observed difference of the dependency of volumetric strain on mean pressure can be related to different factors that differ between experimental and numerical tests:

1. Difference of the compressibility behavior induced by the isotropic compression: for the numerical sample, the relative variations of porosity after compaction when confining pressure is increased from $\mathrm{Pc}=100 \mathrm{kPa}$ to $200 \mathrm{kPa}$, and up to $\mathrm{Pc}=400$ $\mathrm{kPa}$, are $-0.09 \%$ and $-0.81 \%$ respectively, whereas experimentally the sand sample presents a more important compressibility with relative porosity variations equal to $-0.82 \%$ and $-2.22 \%$ (always with respect to the state for $\mathrm{Pc}=100 \mathrm{kPa}$ ). This difference is particularly important in the range of confining pressure $100 \mathrm{kPa}-200 \mathrm{kPa}$ where the relative reduction of porosity for the numerical model is about 9 times smaller than what is found experimentally. This may contribute to the discrepancy between numerical and experimental volumetric strains ob- served, especially for the compression at $\mathrm{Pc}=100$ $\mathrm{kPa}$.

2. Different particle shapes: this is related to the ability for angular or elongated particles to untangle. A reduction of the mean pressure, allowing more flexibility for angular or elongated particles to rotate, can promote their disentanglement, and thus a lower contractive behavior. In addition, even without any translation motion of particles, new contacts may be formed with elongated particles in rotation, limiting further compaction of the granular assembly. Such mechanisms are not reproduced with the numerical model made of spheres, even with the introduction of a contact resistance to rolling, and apparently, constitutes a limitation of the model.

\section{Conclusion}

This paper presented the DEM modeling of the soil mechanical behaviors by using spherical particles and contact rolling resistance. It was demonstrated that the macroscopic behavior of the granular assembly mainly depends, on one hand, on the initial packing density and, on the other hand, on the model parameter fixing the plastic rolling moment at contacts. Other parameters as normal contact stiffness or contact friction angle prove to play a secondary role (bearing in mind that the contacts should be sufficiently stiff and based on a given particle gradation). This is consistent with the fact that the shear strength in cohesionless soil is more strongly related to particle shape (presented here by the plastic rolling moment) than to the inter-particle friction angle, as shown previously by [10]. Moreover, it is greatly affected, together with dilatancy, by the initial density. Consequently, a new numerical method of compaction was proposed, involving either the introduction of interparticle adhesion forces or the lubrication of contacts, to reach a wide range of initial density of granular assemblies, from very loose to very dense. However, it was shown that even if our model succeeds in reproducing the experimental data, there are some limitations to be taken into account related to the description of the dependency of volumetric strains on the mean pressure. This is due to the difference in the relative variation of 

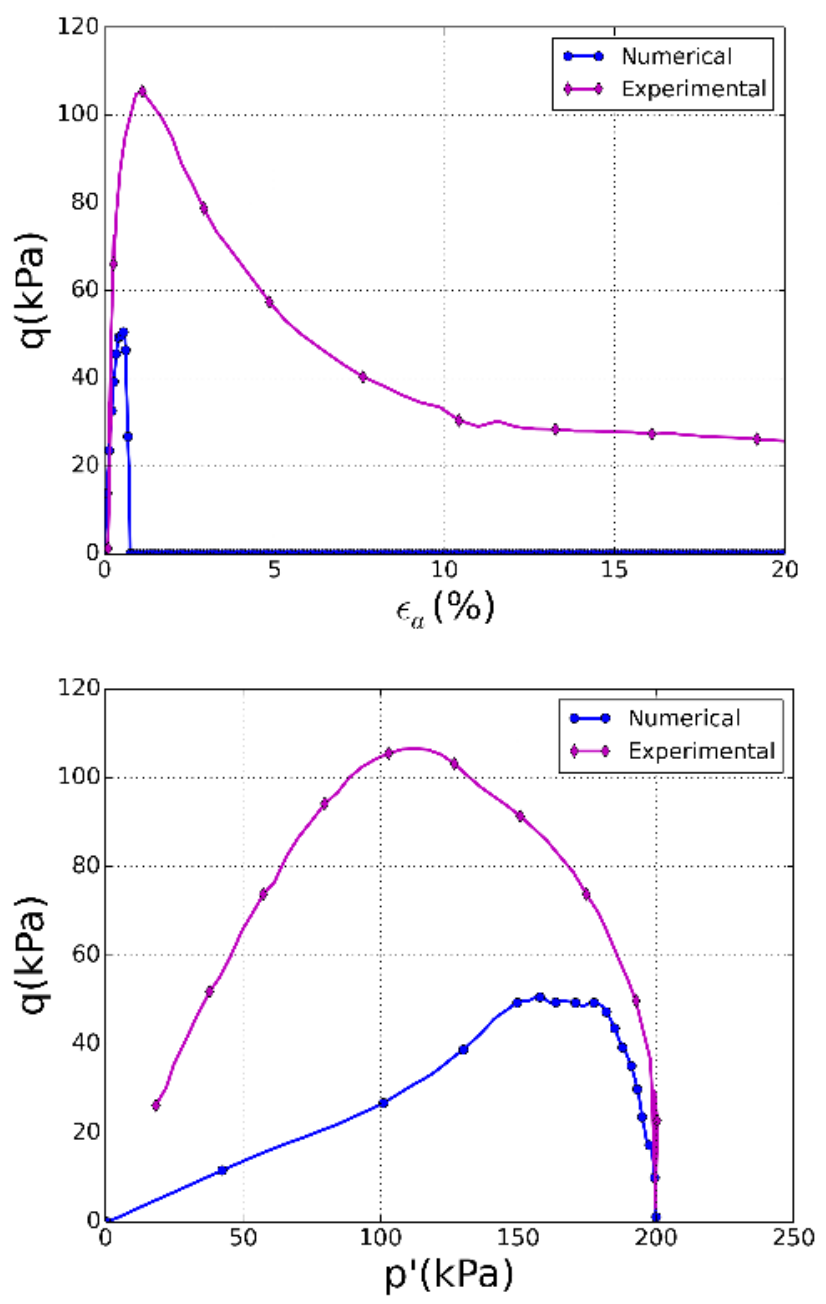

Fig. 11: Comparison between experimental and numerical responses under undrained triaxial compression.

initial porosity after compaction between experimental and numerical tests as well as due to the different particle shapes. Such marked differences may be partially due to the very loose state of the tested sample.

Despite the latter limitation, one should keep in mind the little number of parameters involved in such a model. In total five parameters are involved, among which three elastic parameters are secondary if sufficiently close to the stiff limit, compared to a basic phenomenological elasto-plastic constitutive relation (without any hardening mechanisms) involving a minimum of four parameters (Young modulus, Poisson ratio, internal friction angle, and dilatancy angle). Moreover, the dependency of the dilatancy angle or the friction angle on the mean pressure for such elasto-plastic constitutive relation requires some additional empirical laws coming with additional parameters.
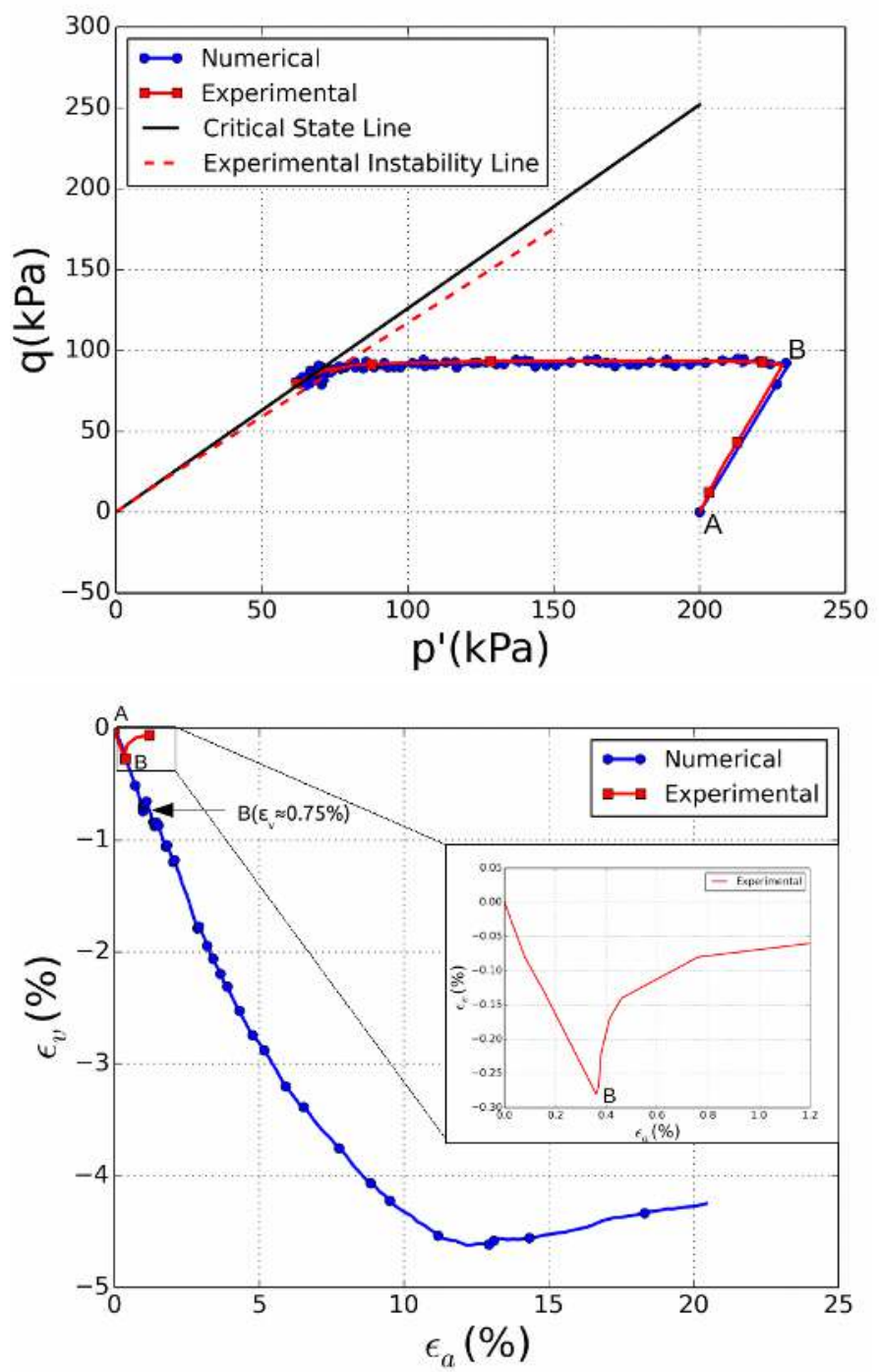

Fig. 12: Comparison between experimental and numerical responses under constant deviator stress loading path just up to failure point.

\section{Acknowledgements}

The environment and support provided by the French research group GDR MeGe 3176 is gratefully acknowledged.

Laboratory 3SR is part of the LabEx Tec 21 (Investissements d'Avenir - grant agreement $n^{\circ}$ ANR-11-LABX0030)

\section{Appendix}

Let $\vec{u}, \vec{\theta}$ define small movements of a particle relative to an initially stable configuration in which the total force and torque on the particle are $\vec{F}=\vec{T}=\overrightarrow{0}$. If the particle is involved in $N_{c}$ contacts and every contact remains elastic (worst case), the 
new force and torque induced by $\vec{u}$ and $\vec{\theta}$ can be expressed as follows, in which the translational and the rotational degrees of freedom are uncoupled (this uncoupling assumption is also found in [12]): (Note that since $\vec{F}=\vec{T}=\overrightarrow{0}$, then we can write $\Delta \vec{F}=\vec{F}$ and $\Delta \vec{T}=\vec{T})$ :

$\vec{F} \simeq-\left(\sum_{c=1}^{N_{c}}\left(K_{n}-K_{s}\right)(\vec{n} \otimes \vec{n})+K_{s} \mathbf{I}\right) \vec{u}$

$\vec{T} \simeq-r^{2} \sum_{c=1}^{N_{c}} K_{s}(\mathbf{I}-\vec{n} \otimes \vec{n}) \vec{\theta}$

I being the inertia tensor of the particle and $r$ its radius. These forces are given by neglecting the rotational term in equation 12 and the translational term in equation 13.

If contact moments $\left(\vec{M}_{r}\right.$ and $\left.\vec{M}_{t w}\right)$ are present, the moments (initially null) induced at each contact (remaining elastic) by $\vec{u}$ and $\vec{\theta}$ are:

$$
\begin{aligned}
\vec{M}_{t w} & =-K_{t w}(\vec{\theta} \cdot \vec{n}) \vec{n} \\
& =-K_{t w}(\vec{n} \otimes \vec{n}) \vec{\theta},
\end{aligned}
$$

$$
\begin{aligned}
\vec{M}_{r} & =-K_{r}(\vec{\theta}-(\vec{\theta} \cdot \vec{n}) \vec{n}) \\
& =-K_{r}(\mathbf{I}-\vec{n} \otimes \vec{n}) \vec{\theta} .
\end{aligned}
$$

where $K_{r}$ and $K_{t w}$ are the rolling and twisting stiffnesses, respectively. Note that, in our study, only the rolling moment was taken into consideration. However, for generalization, we added the twisting moment here.

Adding these contributions (eq. 14 and 15) to the global rotational stiffness (eq. 13) yields

$\vec{T} \simeq-\sum_{c=1}^{N_{c}}\left\{r^{2} K_{s}(\mathbf{I}-\vec{n} \otimes \vec{n})+K_{t w} \vec{n} \otimes \vec{n}+K_{r}(\mathbf{I}-\vec{n} \otimes \vec{n})\right\} \vec{\theta}$

The uncoupled equations of motion for the particle can be expressed as:

$\vec{u}=-\frac{\mathbf{K}_{u}}{M} \vec{u}$,

$\vec{\theta}=-\frac{\mathbf{K}_{\theta}}{J} \vec{\theta}$,

where $\mathbf{K}_{u}$ and $\mathbf{K}_{\theta}$ are the tensors defined by equations 12 and 16 respectively, $M$ is the mass of the particle, and $J$ is the moment of inertia (equal to $\frac{2}{5} r^{2} M$ for a sphere).

$\frac{\mathbf{K}_{u}}{M}$ and $\frac{\mathbf{K}_{\theta}}{J}$ are symmetric positive definite tensors from which six eigen values can be calculated, corresponding to six natural frequencies of the particle. The explicit centered finite difference scheme is stable for the above equations - for a single particle $b$ - as long as $\Delta t$ is less than $2 / \sqrt{\lambda_{\max }^{b}}, \lambda_{\max }^{b}$ being the maximum eigenvalue of the particle.

A multi-body system as a whole may have natural oscillation modes combining the motion of multiple particles, with frequencies higher than the maximum frequency found for individual particles. An accurate determination of the critical timestep would thus need to consider the stiffness matrix of the system as a whole where the motion of two particles in contact would be included. For $N_{b}$ particles it would lead to a matrix of size $\left(6 N_{b}\right)^{2}$ from which eigenvalues would have to be extracted. It can be avoided by remarking that this large assembled matrix would have every contact stiffness appearing twice in each row (once in the diagonal term and once in the column whose index corresponds to the other particle forming the contact). Since the absolute row-sum of the components can be used as an upper bound of the maximum eigenvalue (Perron-Froebenius theorem [3]) an upper bound is obtained simply by multiplying every stiffness by two when calculating the frequencies of one single particle. Equivalently, we can keep the stiffness unchanged and define the upper bound using the eigenvalues associated to individual particles as

$\lambda_{\max }<2 \max _{b}\left(\lambda_{\max }^{b}\right)$.

[13] suggested a similar inequality without proof. Finally, a sufficient condition of stability is:

$\Delta t<\sqrt{\frac{2}{\max _{b}\left(\lambda_{\max }^{b}\right)}}$.

The equations of motion for a particle, 17 and 18, can be written in a simplified way considering the following assumptions:

1. all spheres are identical (same size and same inertia)

2. all contacts have the same stiffness values

3. the stiffness tensors, defined by equations 12 and 16 , are isotropic.

We note also that $K_{r}=\alpha_{r n} r^{2} K_{n}$ and $K-t w=\alpha_{t w} r^{2} K_{n}$ Then the uncoupled equations of motion read:

$\vec{u}=-\frac{N_{c} K_{n}\left(1+2 \alpha_{s}\right)}{M} \vec{u}$,

$\overrightarrow{\ddot{\theta}}=-\frac{N_{c} K_{n}\left(5 \alpha_{s}+5 \alpha_{r n}+2.5 \alpha_{t w}\right)}{M} \vec{\theta}$

\section{References}

1. J.P Bardet. Observations on the effects of particle rotations on the failure of idealized granular materials. $\mathrm{Me}-$ chanics of Materials, 18(2):159-182, 1994.

2. N. Benahmed, T.K. Nguyen, P.Y. Hicher, and M. Nicolas. An experimental investigation into the effects of low plastic fines content on the behaviour of sand/silt mixtures. European Journal of environmental and civil engineering, 19(1):109-128, 2015.

3. A. Brauer and A.C. Mewborn. The greatest distance between two characteristic roots of a matrix. Duke Math J., 26(4):653-661, 1959.

4. F. Calvetti, G. Viggiani, and C. Tamagnini. A numerical investigation of the incremental behavior of granular soils. Riv.Ital.Geotech, 3:11-29, 2003.

5. B. Chareyre, L. Briançon, and P. Villard. Theoretical versus experimental modeling of the anchorage capacity of geotextiles in trenches. Geosynth. Int., 9(2):97-123, 2002.

6. B. Chareyre and P. Villard. Dynamic spar elements and discrete element methods in two dimensions for the modeling of soil-inclusion problems. Journal of Engineering Mechanics, 131(7):689-698, 2005. 
7. G. Combe. Origines géométrique du comportement quasistatique des assemblages granulaires denses: étude par simulations numériques. $\mathrm{PhD}$ thesis, Ecole nationale des ponts et chaussées, 2001.

8. P.A. Cundall and O.D.L. Strack. A discrete numerical model for granular assemblies. Géotechnique, 29(1):4765, 1979.

9. F. da Cruz, S. Emam, M. Prochnow, J-N. Roux, and F. Chevoir. Rheophysics of dense granular materials: Discrete simulation of plane shear flows. Physical review E, 72, 021309, 2005.

10. N. Estrada, E. Azéma, F. Radjaï, and A. Taboada. Comparison of the effects of rolling resistance and angularity in sheared granular media. Powder and grains, hal00842799:891-894, 2013.

11. F. Gilabert, J.-N. Roux, and A. Castellanos. Computer simulation of model cohesive powders: Influence of assembling procedure and contact laws on low consolidation states. Physical Review E, 75, 011303, 2007.

12. Itasca Consulting Group. PFC3D theory and background manual, version 3.0, 2003.

13. R. Hart, P.A. Cundall, and J. Lemos. Formulation of a three-dimensional distinct element model-part II. mechanical calculations for motion and interaction of a system composed of many polyhedral blocks. International Journal of Rock Mechanics and Mining Sciences $\&$ Geomechanics Abstracts, 25(3):117-125, 1988.

14. X. Huang, K.J. Hanley, C. O'Sullivan, and C.K. Kwok. Exploring the influence of interparticle friction on critical state behavior using DEM. Int. J. Numer. Anal. Meth. Geomech, 38(12):1276-1297, 2014.

15. K. Iwashita and M. Oda. Micro-deformation mechanism of shear banding process based on modified distinct element method. Powder Technology, 109(1-3):192-205, 2000 .

16. M.J. Jiang, J.M. Konrad, and S. Leroueil. An efficient technique for generating homogeneous specimens for DEM studies. Computers and Geotechnics, 30(7):579597, 2003.

17. J. Kozicki and J. Tejchman. Numerical simulations of sand behaviour using DEM with two different descriptions of grain roughness. In II International Conference on Particle-based Methods - Fundamentals and Applications, Particles, 2011.

18. J. Kozicki, J. Tejchman, and D. Leśniewska. Study of some micro-structural phenomena in granular shear zones. In Powder and Grains, AIP Conference proceeding, volume 1542, 2013.

19. S.J. Lee, Y.M.A. Hashash, and E.G. Nezami. Simulation of triaxial compression tests with polyhedral discrete elements. Computers and Geotechnics, 43:92-100, 2012.

20. T.K. Nguyen. Etude expérimentale du comportement instable d'un sable silteux: Application aux digues de protection. PhD thesis, Université d'Aix-Marseille, 2014.

21. M. Oda and K. Iwashita. Study on couple stress and shear band development in granular media based on numerical simulation analyses. International Journal of Engineering Sciences, 38(15):1713-1740, 2000.

22. M. Oda, J. Konishi, and S. Nemat-Nasser. Experimental micromechanical evaluation of strength of granular materials: Effects of particle rolling. Mechanics of Materials, 1(4):269-283, 1982.

23. J.-P. Plassiard, N. Belheine, and F.-V. Donzé. A spherical discrete element model: calibration procedure and incremental response. Granular Matter, 11(5):293-306, 2009.

24. F. Radjaï and F. Dubois. Discrete-element modeling of granular materials. ISTE Ltd and John Wiley \& Sons, 2011.
25. J-N. Roux and F. Chevoir. Discrete numerical simulation and the mechanical behavior of granular materials. Bulletin des laboratoires des ponts et chaussées - n 254, 2005.

26. J-N. Roux and G. Combe. How granular materials deform in quasistatic conditions. In IUTAM-ISIMM Symposium on Mathematical Modeling and Physical Instances of Granular Flow. AIP publishing., volume 1227(1), pages 260-270, 2010

27. C. Salot, P. Gotteland, and P. Villard. Influence of relative density on granular materials behavior: DEM simulations of triaxial tests. Granular Matter, 11(4):221-236, 2009.

28. L. Scholtés, B. Chareyre, F. Nicot, and F. Darve. Micromechanics of granular materials with capillary effects. International Journal of Engineering Science, 47(1112):1460-1471, 2009.

29. B. Suhr and K. Six. On the effect of stress dependent interparticle friction in direct shear tests. Powder Technology, 294:211-220, 2016.

30. K. Szarf, G. Combe, and P. Villard. Polygons vs. clumps of discs: A numerical study of the influence of grain shape on the mechanical behaviour of granular materials. Powder Technology, 208(2):279-288, 2011.

31. C. Thornton. Numerical simulations of deviatoric shear deformation of granular media. Géotechnique, 50(1):4353, 2000.

32. JM. Ting, BT. Corkum, CR. Kauffman, and C. Greco. Discrete numerical model for soil mechanics. J Geotech Eng, 115(3):379-398, 1989.

33. A.-T. Tong, E. Catalano, and B. Chareyre. Pore-scale flow simulations: Model predictions compared with experiments on bi-dispersed granular assemblies. Oil $\&$ Gas Science and Technology - Revue IFP Energies nouvelles, 67(5):743-752, 2012.

34. V. Šmilauer et al. Yade Documentation 2nd ed. The Yade Project, 2015. http://yade-dem.org/doc/.

35. X. Wang and Li J. Simulation of triaxial response of granular materials by modified DEM. Science China: Physics, Mechanics and Astronomy., 57(12):2297-2308, 2014.

36. L. Widuliński, J. Kozicki, and J. Tejchman. Numerical simulations of triaxial test with sand using DEM. Archives of Hydro-Engineering and Environmental Mechanics, 56(3-4):149-171, 2009. 